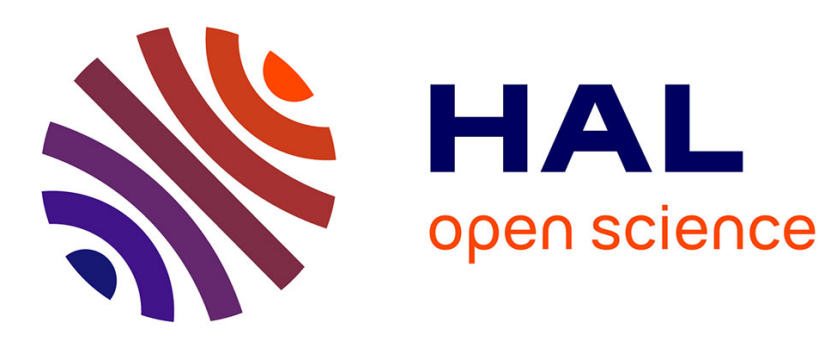

\title{
From equilibrium lamellae to out-of-equilibrium cylinders in triblock copolymer nanolayers obtained via multilayer coextrusion
}

Juan-Sebastian Montana, Sébastien Roland, Emmanuel Richaud, Guillaume Miquelard-Garnier

\section{To cite this version:}

Juan-Sebastian Montana, Sébastien Roland, Emmanuel Richaud, Guillaume Miquelard-Garnier. From equilibrium lamellae to out-of-equilibrium cylinders in triblock copolymer nanolayers obtained via multilayer coextrusion. Polymer, 2018, 136, pp.27-36. 10.1016/j.polymer.2017.12.044 . hal-01825301

\section{HAL Id: hal-01825301 https://hal.science/hal-01825301}

Submitted on 28 Jun 2018

HAL is a multi-disciplinary open access archive for the deposit and dissemination of scientific research documents, whether they are published or not. The documents may come from teaching and research institutions in France or abroad, or from public or private research centers.
L'archive ouverte pluridisciplinaire HAL, est destinée au dépôt et à la diffusion de documents scientifiques de niveau recherche, publiés ou non, émanant des établissements d'enseignement et de recherche français ou étrangers, des laboratoires publics ou privés. 


\title{
From equilibrium lamellae to out-of-equilibrium cylinders in triblock copolymer nanolayers obtained via multilayer coextrusion
}

\author{
Juan-Sebastián Montana, Sébastien Roland**, Emmanuel Richaud, \\ Guillaume Miquelard-Garnier*
}

PIMM, UMR 8006, ENSAM - CNRS - CNAM, HESAM Université, 151 boulevard de l'Hôpital, 75013 Paris, France

\section{A R T I C L E I N F O}

\section{Article history:}

Received 5 September 2017

Received in revised form

17 November 2017

Accepted 17 December 2017

Available online 18 December 2017

\section{Keywords:}

Multilayer coextrusion

Triblock copolymer

Directed self-assembly

\begin{abstract}
A B S T R A C T
Multilayer coextrusion was used to obtain nanolayered films of self-assembled commercial triblock copolymer poly(methyl methacrylate- $b$-butyl acrylate- $b$-methyl methacrylate) (MAM) confined by pol$\mathrm{y}$ (methyl methacrylate) (PMMA). The MAM layer thickness was varied from $30 \mathrm{~nm}$ to $500 \mathrm{~nm}$ (i.e. roughly 1 to $15-20$ nanodomains) by changing either the number of multiplying elements or the chill roll draw ratio. The as-extruded triblock morphology within the layers was identified as cylindrical using transmission electronic microscopy (TEM) and small-angle X-ray scattering (SAXS). Surprisingly, this differs from the lamellar morphology identified at equilibrium in bulk and thin films for this triblock. Moreover, as the triblock layer thickness is decreased, the triblock morphology is constrained into a preferential orientation. Slightly different packings were observed on films with similar layer thicknesses but achieved with different processing routes. This one-step and industrially scalable method allowing long-range control of the self-assembly is of interest for engineering applications with large quantity of materials needed.
\end{abstract}

\section{Introduction}

Block copolymers (BCPs) display an ability to self-assemble [1] Most polymer pairs are immiscible with each other and tend to phase separate when blended together [2]. In the case of BCPs, because of the covalent bonds between the segments, a macrophase separation cannot be reached, and they micro (or rather nano) phase separate, developing well-ordered structures with typical dimensions of a few tens of nanometers [3,4].

Various morphologies can be reached at the thermodynamic equilibrium, according to the well-known phase diagram which depends on the Flory interaction parameter $(\chi)$ and the degree of polymerization $(N)$ of the BCP on one side, and on the volume fraction $(f)$ of the blocks on the other $[3,4]$.

The variety of morphologies theoretically achievable and their typical sizes make BCPs attractive for many applications such as soft nanolithography, nanomembranes, or as toughening agents in

\footnotetext{
* Corresponding author.

** Corresponding author.

E-mail addresses: sebastien.roland@ensam.eu (S. Roland), guillaume. miquelardgarnier@lecnam.net (G. Miquelard-Garnier).
}

nanoblends [5,6]. Most of the applications require a high level of control not only of the morphologies but also of the nanodomains order [5,6]. However, while self-assembling, the nanostructures grow randomly with a periodical order maintained only over some micrometers and develop usually a polygrain structure.

Several intrinsic (such as the polymer architecture, the block symmetry, the conformational asymmetry, the fluctuation parameter or the dispersity), or extrinsic (confinement or shear) factors have been showed to influence the block copolymer morphologies by impacting the phase diagram (i.e. the equilibrium morphology with regard to the copolymer composition) and/or the orientation of the nanodomains, and in some cases by allowing the block copolymers to achieve out-of-equilibrium but still well-defined morphologies.

Amongst the intrinsic parameters, for instance, the architecture of the polymer chain (stars, branched, or comb-like copolymers) influences the shape of the phase diagram [7]. The phase diagram of symmetric triblock copolymers differs from that of a diblock in the Weak Segregation Limit (WSL) resulting in a lower critical $\chi \mathrm{N}$ while the available set of thermodynamic morphologies remains similar [8]. Higher dispersities have also shown to greatly impact the phase diagram [7,9-15], which needs to be accounted for when comparing the morphology of polydisperse BCPs to monodisperse 
analogues. For instance, Matsen [14] predicted through selfconsistent field theory that a 50-50 AB-type diblock copolymer having a dispersity of 2 for block A would not show the expected lamellar morphology. The influence of the dispersity on the morphology has been experimentally confirmed by several studies, for example by Lynd and Hillmyer [11-13]. This may be of importance when working with commercial BCPs synthesized via controlled radical polymerization.

A widely studied extrinsic factor impacting BCP self-assembly is confinement. When the typical size of the confining medium becomes comparable to the BCP size or the nanodomain size, interfacial interactions between the $\mathrm{BCP}$ and the substrate can influence the equilibrium morphology, with new ones possibly arising over the bulk ones [16-19], and nanodomains orientation [20,21].

Imposing shear rate to the BCPs during self-assembly is also known to influence the orientation of the morphology [22-26], as well as the morphology itself [27,28], especially in the vicinity of the order-disorder transition temperature $\left(\mathrm{T}_{\text {odt }}\right)$. For instance, at low $\chi \mathrm{N}(\sim 20)$ and centered $f=0.6$, the modulated (HML) and perforated (HPL) layered structures with hexagonal symmetry have been observed [29]. These metastable morphologies appear to be sensitive to shearing and thus transform into out-ofequilibrium cylinders oriented in the flow direction when heated and sheared [30]. These shear-induced morphology changes have also been observed for gyroid morphology (into cylinders) [31,32] and cylinders into body-centered cubic (BCC) spheres [33,34].

Various methods, such as solvent or temperature annealing sometimes combined with shearing or other external fields (electric, magnetic...), are commonly employed efficiently to create long-range ordering in thin films or in the bulk but are slow and potentially complex or costly as far as industrial applications are concerned or large amounts of materials needed [5,35-37]. In this study, we look at both confinement and shear rates effects on the morphology and orientation of a disperse symmetric lamellar BCP, poly(methyl methacrylate- $b$-butylacrylate- $b$-methyl methacrylate) (MAM) confined by PMMA through an innovative process, nanolayer coextrusion, without additional thermal annealing (i.e., in an out-of-equilibrium state).

Multilayer coextrusion is derived from coextrusion but allows the fabrication of films made of thousands of alternating layers of different polymers with thickness theoretically tuned down to a few tens or even a few nanometers (hence, this process may be named nanolayer coextrusion). The polymers flows pass through a series of layer multiplying elements (LME) which each multiplies the number of layer by two (based on the so-called baker's transformation). This processing tool has been mainly developed by Baer to combine immiscible polymer pairs into unique multilayer structures [38-41]. Korley used it to confine a symmetric triblock, poly(styrene- $b$-ethylene-co-propylene- $b$-styrene) (SEPS) having a rubbery middle block and a cylindrical morphology with two different confiners, namely polystyrene (PS) and poly(methyl methacrylate) (PMMA) [42-44]. In their work, the number of layers remained constant (257) but the layer thickness changed [45]. The authors also showed that these BCPs could be extruded below their $\mathrm{T}_{\text {odt }}$, i.e. with their elastic contribution higher than their viscous one. The resulting materials displayed anisotropic mechanical properties due to a higher degree of orientation of the cylinders in the extrusion direction. Their work also showed that the packing of the cylinders was improved with the reduction of the layer thickness, especially after long times annealing [42-44]. A last article showed similar results for a spherical triblock (SEBS) extruded above $\mathrm{T}_{\text {odt }}$ [46].

Nanolayer coextrusion has been developed in our lab [47-50] and also used recently to confine an asymmetric BCP poly(styrene- $b$-butadiene- $b$-methyl methacrylate) (SBM) with an incompatible matrix (polycarbonate PC) [51]. It was shown that as the triblock layer thickness decreases and the draw ratio increases, the triblock morphology is constrained into a preferential orientation and higher long-range order is observed [51].

In this study, the effect of processing parameters on the resulting morphologies has been studied. Long-range order within the confined layers is achieved (and can be to some extent controlled via the processing parameters) on large scales of material through an industrially relevant process, similar to previous studies [42-44,46,51]. More surprisingly and rarely observed experimentally (and to the best of our knowledge for the first time in a materials engineering context), it is shown that nanolayer coextrusion favors through shearing and "soft" confinement a cylindrical morphology over a lamellar one more thermodynamically favored. This morphology is clearly defined despite the short processing time, with a degree of ordering improved with increased confinement. It is also observed that similar layer thicknesses obtained through different processing routes lead to slightly different packings of the cylinders. This study shall be of help for applications such as mechanical toughening where a control of the morphology is necessary but difficult to achieve with classical processing tools.

\section{Materials and methods}

\subsection{Materials}

Poly(methyl methacrylate-b-butyl acrylate-b-methyl methacrylate) (PMMA-PBA-PMMA or simply MAM) lamellar triblock copolymer was gratefully supplied by Arkema from their Nanostrength ${ }^{\circledR}$ trademark, under the reference M53, and used as received without further purification. Average molecular weights $M_{w}=92.5 \mathrm{~kg} / \mathrm{mol}$, $M_{n}=43.2 \mathrm{~kg} / \mathrm{mol}$ and dispersity $\bigoplus_{\mathrm{M}}=2.14$ were verified by a high temperature size exclusion chromatography SEC (PL-GPC $220^{\circledR}$ from Agilent Technologies) calibrated with polystyrene Shodex ${ }^{\circledR}$ standards, using 1,2,4- trichlorobenzene (Chromasolv, SigmaAldrich) at $135^{\circ} \mathrm{C}$ and a flow rate of $1 \mathrm{~mL} / \mathrm{min}$. The triblock composition was obtained via ${ }^{1} \mathrm{H}$ NMR spectra in $\mathrm{CDCl}_{3}$ with 32 scans on a Bruker apparatus at $300 \mathrm{MHz}$ (data not shown). A final composition of $23-54-23$ wt\% was obtained, in good agreement with the supplier information (23.5-53-23.5 wt\%, i.e. a volume fraction of 56\% of PBA and 44\% of PMMA using the densities of the two polymers [9]), which should lead to a lamellar morphology. The $T_{\text {odt }}$ can be identified by rheology as a critical temperature at which the value of the storage modulus $\left(G^{\prime}\right)$ drops precipitously. The MAM T $\mathrm{T}_{\text {odt }}$ was higher than $240^{\circ} \mathrm{C}, \mathrm{G}^{\prime}$ being higher than the loss modulus $\left(\mathrm{G}^{\prime \prime}\right)$ during the whole frequency sweep.

Poly(methyl methacrylate) VM100 was purchased from Altuglas International and used (as received) as a confining polymer. Its melt flow index (MFI) is $14.5 \mathrm{~g} / 10 \mathrm{~min}\left(230^{\circ} \mathrm{C} / 3.8 \mathrm{~kg}\right)$ as provided by Altuglas International. The molecular weight $M_{w}=139 \mathrm{~kg} / \mathrm{mol}$ and dispersity $\bigoplus_{\mathrm{M}}=2.1$ were obtained using a Waters $717+$ GPC at $35^{\circ} \mathrm{C}$ with PMMA standards and THF (Alfa-Aesar, purity: $99 \%$ ) as an eluent. The PMMA grade was chosen so the two polymers have similar viscosities at the temperature process $\left(225^{\circ} \mathrm{C}\right)$ and shear rates $\left(3-20 \mathrm{~s}^{-1}\right)$. In this range of shear rates, assumed to be those in the LMEs, the viscosity ratio remains between 0.51 and 0.84 , as measured with an Anton-Paar MCR 502 rheometer via a frequency sweep test (strain amplitude fixed at $1 \%$ ) at the extrusion temperature under a nitrogen atmosphere (see supporting information, figure SI-1).

Prior to extrusion, MAM and PMMA VM100 pellets were separately dried in a SOMOS dry air T20 eco system at $80^{\circ} \mathrm{C}$ for $24 \mathrm{~h}$. 


\subsection{Films preparation}

\subsubsection{Nanolayer coextrusion}

Films were fabricated using LMEs at the end of a classical trilayer coextrusion set-up with MAM as the middle layer of the trilayer flow and PMMA as outer layers. The processing route consists of two single screw extruders, a 3-layer coextrusion feedblock (ABA), LMEs in a series disposition, a flat die-end film, and a thermally regulated chill roll. A $20 \mathrm{~mm}$-diameter Scamex extruder was used for each polymer, set to $90 \%$ PMMA for $10 \%$ MAM (wt\%) by adjusting the screw speeds of each extruder to control the throughput. This proportion was somewhat arbitrarily chosen but allows varying the confined layers thicknesses between a few tens and a few hundreds of nm by varying either the LMEs and/or the draw ratio within reasonable ranges. The whole set-up (extruders, feedblock, and LMEs) temperature was set to $225^{\circ} \mathrm{C}$ i.e. below the MAM $T_{\text {odt }}$. The theoretical number of alternating layers $(\mathrm{N})$ in the film is determined by the number of LMEs $(n)$ from the equation $\mathrm{N}=2^{(\mathrm{n}+1)}+1$. Depending on the targeted thickness of the confined MAM layers, 8 (513 total layers) or 9 (1025 total layers) LMEs were used. After the flat die, the films were drawn with a chill roll at $80{ }^{\circ} \mathrm{C}$. Draw ratio (Dr) is defined as the ratio of the chill roll speed over extrusion flow rate at flat die and ranges from 1 to 10 (with a chill roll speed ranging from 80 to $1000 \mathrm{~cm} / \mathrm{min}$ ) (see Fig. 1). The typical total film thickness ranges from $100 \mu \mathrm{m}$ (high Dr) to $1 \mathrm{~mm}$ (low Dr), while the width is on the order of $10 \mathrm{~cm}$ and the length is in the meter range (actually the length is only dependent on the fact that extruders are being fed with polymer pellets).

Additional samples (pure MAM and PMMA/MAM 90/10 wt\% dry blends) were obtained through conventional single-screw extrusion at $225^{\circ} \mathrm{C}$ to characterize the as-extruded MAM morphology in bulk and blends.

\subsubsection{Solvent cast}

Thick film specimens of MAM triblock copolymer were prepared by casting from chloroform solution followed by slow solvent evaporation at room temperature to study the bulk equilibrium morphology. A $10 \mathrm{wt} \%$ solution of MAM in chloroform was poured in an aluminum dish covered with a glass plate to ensure a slow evaporation ( 6 days). Films with a thickness of $\sim 740 \mu \mathrm{m}$ were thus obtained. The films were then cryo-sectioned perpendicular to their surface with an ultramicrotome Leica EM FC6 at $-100{ }^{\circ} \mathrm{C}$, equipped with a Diatome diamond knife at a cutting rate of $1 \mathrm{~mm} / \mathrm{s}$. AFM images were then taken on the prepared surfaces.

\subsubsection{Spin-coating}

MAM films of 45 and $110 \mathrm{~nm}$ thickness were obtained with a SPS 150 Europe spin-coater. Silicon wafers (2 inches diameter from Sil'tronix silicon technologies) were used as substrate, and MAM solutions in toluene at $1.5 \mathrm{wt} \%$ and $2 \mathrm{wt} \%$ were respectively spincoated at $3000 \mathrm{rpm}$ and $1100 \mathrm{rpm}$ with a fixed acceleration of $4000 \mathrm{rpm} / \mathrm{s}$. The films were then annealed under vacuum up to $72 \mathrm{~h}$ at $170^{\circ} \mathrm{C}$, a temperature between the highest $\mathrm{T}_{\mathrm{g}}$ block $\left(97^{\circ} \mathrm{C}\right)$ and $\mathrm{T}_{\text {odt }}$ (above $240^{\circ} \mathrm{C}$ ), to characterize the confined equilibrium morphology. AFM images were taken at different annealing times to observe the MAM nano-structuration evolution.

\subsection{Atomic force microscopy (AFM)}

AFM tapping mode images were obtained by using a multimode microscope driven by a Nanoscope V controller (Veeco) and operated under ambient atmospheric conditions. The tips (silicon, spring constant $40 \mathrm{~N} / \mathrm{m}$, oscillation frequency ca. $300 \mathrm{kHz}$ ) were supplied by BudgetSensors. The tips have a curvature radius below $10 \mathrm{~nm}$. Topographic, amplitude, and phase images are acquired simultaneously, the last one allowing the micro-phase separation of each polymer block to be identified. Images were recorded with a resolution of $512 \times 512$ pixels and at a scan rate of $0.8 \mathrm{~Hz}$. These settings yield a pixel size of $4 \mathrm{~nm}$. In phase images, confining PMMA appears in gold and confined MAM appears in brown with golden spots corresponding to the PBA and PMMA blocks, respectively.

\subsection{Transmission electron microscopy (TEM)}

2 TEM microscopes have been used to study the morphology of MAM on the extruded films in the $\mathrm{x}$ axis (extrusion direction, ED)

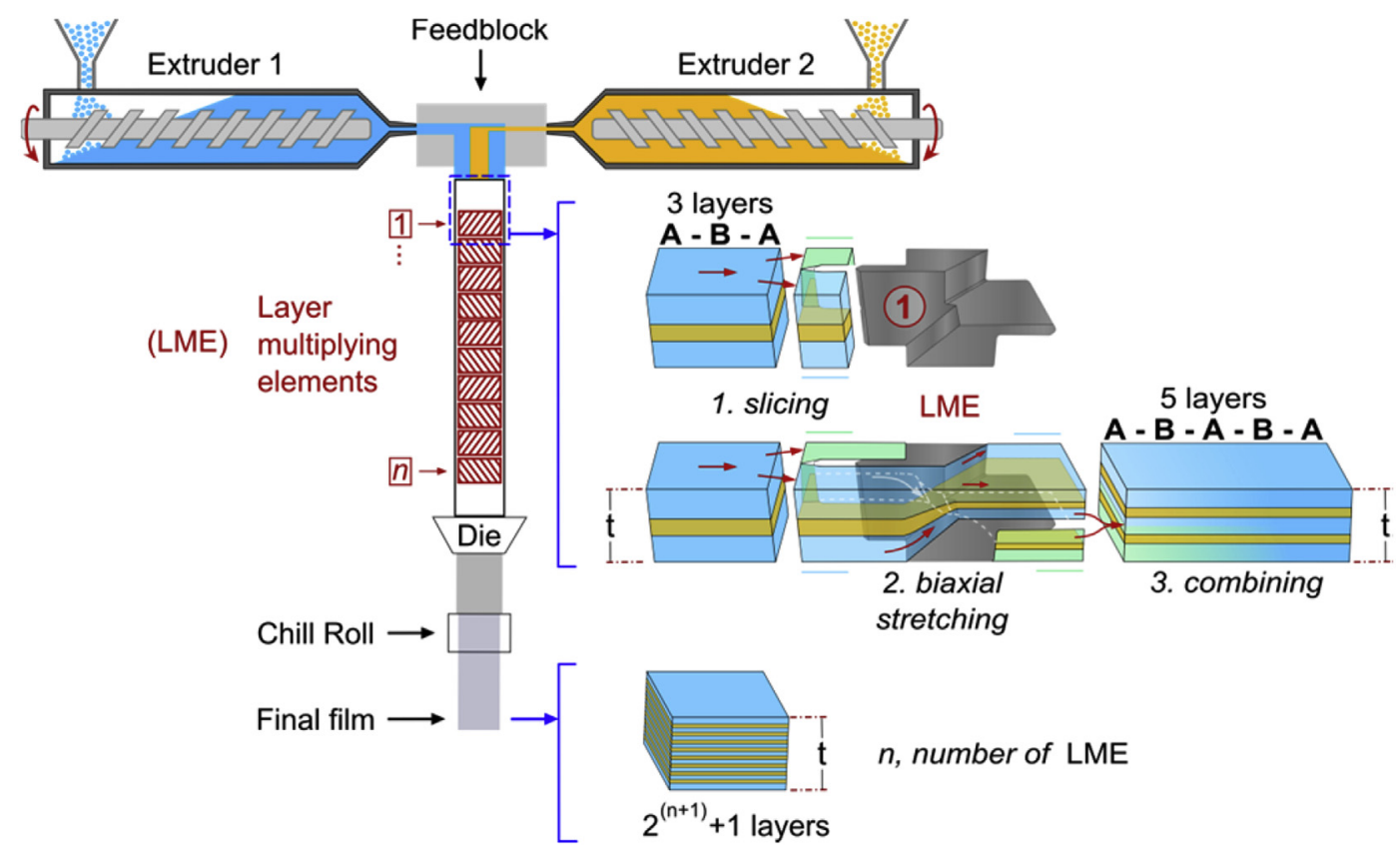

Fig. 1. Schematic of the nanolayer coextrusion setup. 
and in the $y$ axis (lateral direction, $L D$ ), so the electron beam is parallel and perpendicular to the ED, respectively. The first microscope is a Zeiss $912 \Omega$ operated at an accelerated voltage of $80 \mathrm{kV}$ equipped with a side-mounted $2 \mathrm{k} \times 2 \mathrm{k}$ Veleta CDD camera (Olympus), and the second one is a TEM JEOL JEM-2100 operated at an accelerated voltage of $200 \mathrm{kV}$ equipped with a post-column spectrometer (GIF-Tridiem, Gatan), controlled by Digital Micrograph software (versions 1.83.842, Gatan). Prior to the observation, the pre-faced samples of extruded films were immerged in a $1 \%$ $\mathrm{RuO}_{4}$ solution produced in situ by combining sodium hypochlorite ( $\mathrm{NaClO})$ with ruthenium(III) chloride $\left(\mathrm{RuCl}_{3} \mathrm{xH}_{2} \mathrm{O}\right)$ (both from Sigma-Aldrich) for $6 \mathrm{~h}$. Ruthenium tetroxide stains preferentially the PBA block, giving darkest colors in the TEM analysis [52]. The PMMA is less reactive to $\mathrm{RuO}_{4}$ and appears transparent, thus brighter in the images. The PBA rubbery phase is crosslinked by staining which allows cutting at room temperature [53]. The stained samples were then sectioned perpendicular to their surface with an ultramicrotome 2088 Ultrotome V (LKB) equipped with a Diatome diamond knife at a cutting rate of $1 \mathrm{~mm} / \mathrm{s}$. Thin slices of 70-80 nm were obtained and placed on TEM grids for observation. At least 15 images were collected from different slices for each film, at different magnifications (from $\times 2400$ leading to image sizes of $25-100 \mu \mathrm{m}^{2}$ to $\times 10600$ leading to image sizes of $0.04-0.25 \mu \mathrm{m}^{2}$ ).

\subsection{Small angle X-ray scattering (SAXS)}

SAXS measurements of the extruded films in the $\mathrm{x}, \mathrm{y}$ (extrusion and lateral directions respectively, as defined in the previous section), and $z$ (transverse direction, TD) axes were performed on the high brilliance SWING beam line at the Soleil Synchrotron facility, with a monochromator set at $12 \mathrm{keV}$ [54]. Using a CCD detector at $2 \mathrm{~m}$ from the sample, diffraction patterns were recorded for reciprocal spacing $\mathrm{q}=4 \pi \sin (\theta) / \lambda$ varying between 0.005 and $0.4 \AA^{-1}$, i.e. repetitive distances $d=2 \pi / q$ ranging from 1256 to $15 \AA$. By using Foxtrot software, 1D SAXS curves were obtained by circular averaging of the full 2D images $\left(0-360^{\circ}\right)$, and for some nonisotropic patterns by averaging $\pm 45^{\circ}$ of the horizontal and vertical axis. 10 images were recorded for each sample and 1D curves were averaged in order to obtain 1 curve for each sample.

Complementary experiments were obtained with a lab SAXS system from Xenocs. The apparatus consists of an X-ray source using a copper anode $K \alpha$ radiation, the wavelength being $1.54 \AA$, a monochromator, a collimator, a specimen chamber, a MAR300 2D detector, and a computer equipped with the MAR300 software. The sample-to-detector distance was $1252 \mathrm{~mm}$. Silver behenate standard reference material was used for sample-to-detector distance calibration. The exposure time was $15000 \mathrm{~s}$. The 2D patterns were also treated using Foxtrot software. The 2D X-ray diffraction patterns were integrated along the azimuthal direction from isotropic samples.

\section{Results}

Let us first characterize the MAM equilibrium morphology both in bulk and thin films.

MAM bulk equilibrium morphology obtained from long annealing of solvent cast films (slow evaporation of the nonselective solvent) is then shown in Fig. 2a (and a'). It reveals a majority of isotropic growing lamellae cut parallel to their normal direction (golden strokes in the figure). The corresponding SAXS pattern can be found in supporting information (figure SI-2). This 1D profile shows slight Bragg peaks at relative positions of $1 q, 2 q$, and $3 q$, suggesting also a lamellar disposition.

The confined MAM equilibrium morphology in spin-coated thin films with $45 \mathrm{~nm}$ and $110 \mathrm{~nm}$ thicknesses annealed for $72 \mathrm{~h}$ at $170^{\circ} \mathrm{C}$ under vacuum is shown in Fig. $2 \mathrm{~b}$ (and $2 \mathrm{~b}^{\prime}$ ) and $2 \mathrm{c}$ (and $2 \mathrm{c}^{\prime}$ ). $72 \mathrm{~h}$ annealing under vacuum was chosen for the characterization of the equilibrium morphology but note that after roughly $12 \mathrm{~h}$, the AFM images at different times do not display significant differences with each other. The 45-nm film shows, as in the bulk, a majority of lamellae, in this case oriented perpendicular to the surface, along with isolated point defects, which can be attributed to disclinations and dislocations [55,56]. The length of the vertical lamellae (strokes) can also be greatly impacted by the dispersity, which is known to influence the interfacial curvature [11], and thus, the defect density [57,58]. For this industrial copolymer which shows relatively high dispersity, it is not surprising to observe short length strokes. The thicker film (Fig. 2c) shows the typical island-and-hole morphology encountered with lamellar block copolymers [5], when the thickness is incommensurate with the lamellar period of the block copolymer. The transition region between two different heights of oriented parallel-at-surface lamellae shows well-defined perpendicular-at-surface lamellae to accommodate the transient step. Even though it is often observed that lamellar diblock copolymer would favor parallel lamellae in thin films to accommodate the substrate/film interface, the perpendicular orientation would appear when triblock analogues are used, especially if the surface energies of the blocks are similar [59].

According to these results, we can assume that the studied MAM copolymer shows mainly a lamellar morphology. We may also note that, using an estimated value for $\chi_{\text {MAM }}(\sim 0.03)$ [9] a $\chi \mathrm{N}$ close to 10 is obtained, placing the MAM close to the lower limit of $\chi \mathrm{N}$ where metastable states (such as HML and HPL morphologies) have been observed experimentally [29].

We will now discuss the as-extruded, without annealing, out-ofequilibrium morphologies obtained in the nanolayer films (due to the extrusion shear flows and relatively fast cooling using the chill rolls). MAM was successfully coextruded below its $\mathrm{T}_{\text {odt }}$ with PMMA as a confiner. Continuous layers were obtained with different thicknesses by varying both the number of LME and Dr. The theoretical MAM layer thickness can be determined knowing the total thickness of the film (varied by changing the draw ratio), the volume fraction of the two polymers and the number of MAM layers (varied by changing the number of LMEs), according to the equation:

$t_{\text {th MAM }}=t_{\text {film }} \times \frac{v o l \%_{\text {MAM }}}{n_{\text {MAM layers }}}$

Films with targeted theoretical thicknesses varying from 30 to $500 \mathrm{~nm}$ for the confined layers were prepared. The mean experimental layer thickness has been obtained by measuring roughly 5-10\% of the MAM layers within the films either using AFM (Fig. 3a) or TEM (Fig. 3b).

The results are summarized in Table 1 . The mean experimental thicknesses varied from 30 to $470 \mathrm{~nm}$, with values within $11 \%$ of the targeted theoretical ones. This small deviation between mean experimental and theoretical layer thickness may either be due to slight changes in the extruders throughput during extrusion (which will modify the film composition) and/or to the sampling size (i.e. the number of layers measured in the sample compared to the total number of layers) used to determine the mean, as discussed in a previous study [60]. Still, there is a satisfactory agreement between the (targeted) MAM theoretical layer thickness and the mean experimental one for all the samples studied. Then, some heterogeneity in terms of individual layer thicknesses within the sample (standard deviation from the mean in the 15-20\% range except for the layers thicker than $250 \mathrm{~nm}$ where standard deviation is close to $30 \%$ ) has been observed (see also Fig. 3) and was attributed by Korley [42] to the effects of elasticity of the BCPs extruded below 

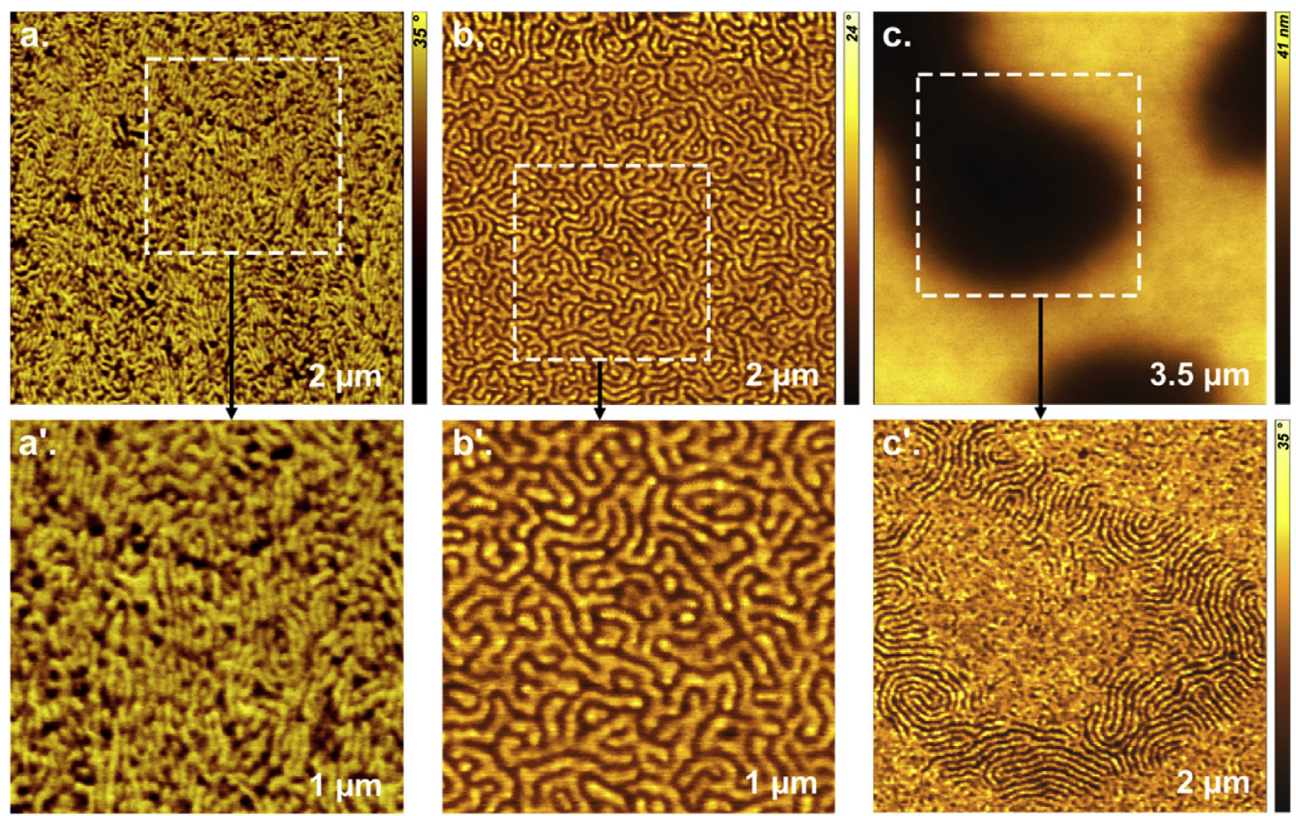

Fig. 2. AFM phase images of MAM morphologies of annealed spin-coated thin films of (a) $45 \mathrm{~nm}$ and (b, topographic image) $110 \mathrm{~nm}$ of thickness; and (c) of a thick annealed solvent cast film. (a'), (b') and (c') are respectively zooms on the white dashed line squares regions.

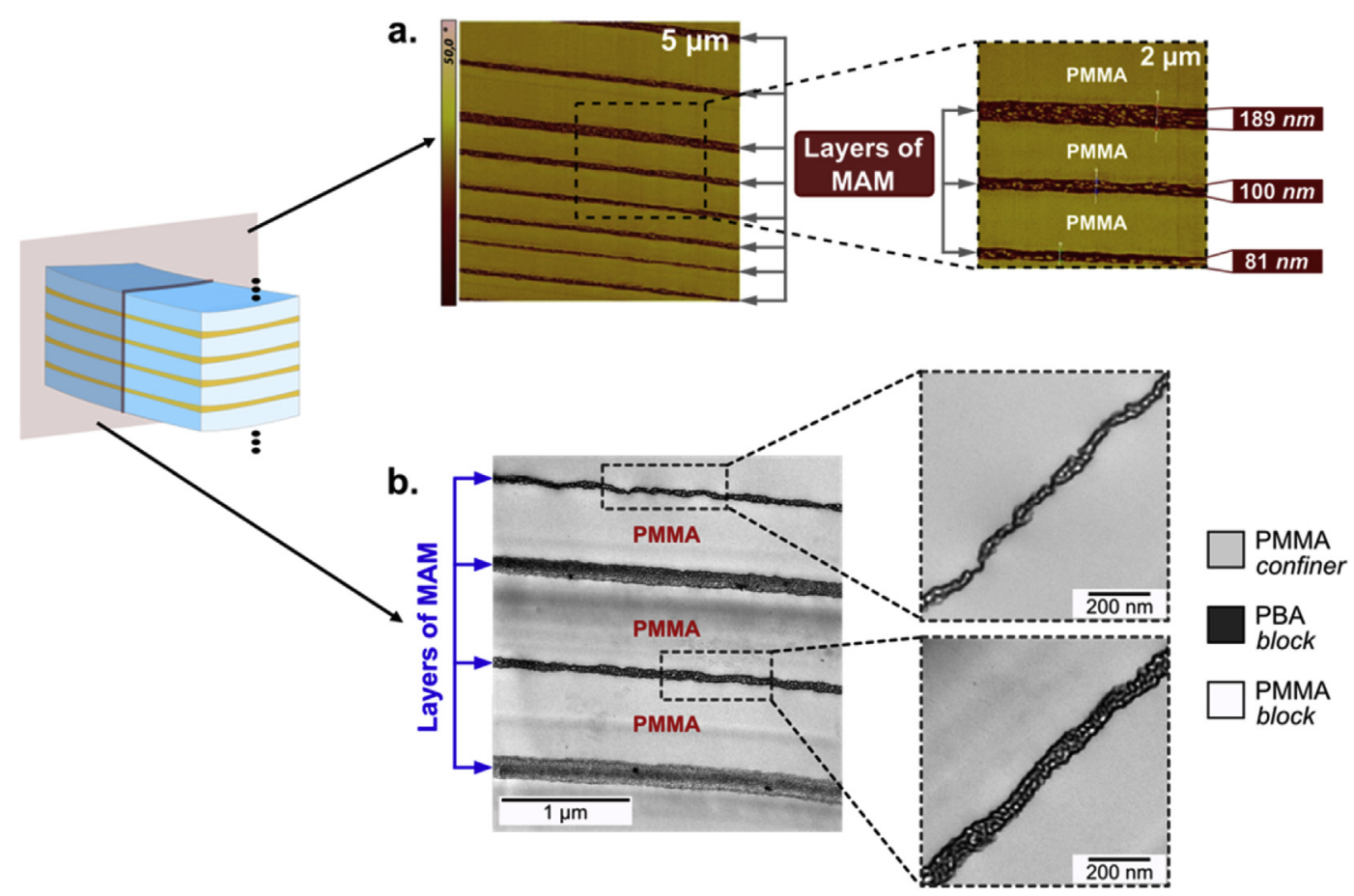

Fig. 3. (a) Phase AFM and (b) TEM images of cross section of a nanolayered film of MAM confined by PMMA.

Todt.

Due to the rubbery phase, sample cutting at room temperature did not allow the observation of the triblock morphology within the layers via AFM. However, staining crosslinks the rubbery phase (see Materials \& Methods) which makes it possible to characterize these morphologies with TEM (see Fig. 3).

The triblock morphologies of the as-extruded films (without annealing, i.e. in an out-of-equilibrium state) in the confined layers were then closely observed by TEM (Fig. 4 and figure SI-3).
Strikingly, a cylindrical morphology (PMMA cylinders) oriented in the extrusion direction can be identified (white dots in the extrusion direction, white lines in the lateral direction). Few articles report such shear-induced transition that could be favored by the high dispersity, which induces interfacial curvature [9]. HPL and HML morphologies are sensitive to shearing and would favor the appearance of cylinders, especially close to the order-disorder transition [30,31,61]. The cylinders are more oriented near the edges of the PMMA layers (i.e. at the interface between MAM and 
Table 1

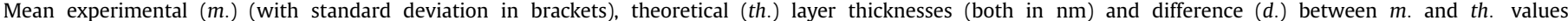
$((m .-t h) /.(t h) \times 100)$.$) for all multilayer samples (90-10 w t \%)$.

\begin{tabular}{|c|c|c|c|c|c|c|c|c|c|c|c|c|c|c|c|}
\hline \multirow[t]{3}{*}{ LME } & \multicolumn{15}{|c|}{$\mathrm{Dr}$} \\
\hline & \multicolumn{3}{|l|}{1} & \multicolumn{3}{|l|}{2} & \multicolumn{3}{|l|}{4} & \multicolumn{3}{|l|}{8} & \multicolumn{3}{|l|}{10} \\
\hline & $m$. & th. & $d(\%)$ & $m$ & th. & $d(\%)$ & $m$ & th. & $d(\%)$ & $m$. & th. & $d(\%)$ & $m$ & th. & $d(\%)$ \\
\hline 8 & $\begin{array}{l}471 \\
(162)\end{array}$ & 527 & 10.7 & $\begin{array}{l}296 \\
(53)\end{array}$ & 316 & 6.4 & $\begin{array}{l}168 \\
(27)\end{array}$ & 186 & 9.2 & $\begin{array}{l}117 \\
(21)\end{array}$ & 127 & 8.1 & $\begin{array}{l}91 \\
(18)\end{array}$ & 82 & 10.9 \\
\hline 9 & $\begin{array}{l}264 \\
(87)\end{array}$ & 269 & 2.0 & $\begin{array}{l}158 \\
(28)\end{array}$ & 145 & 8.9 & $\begin{array}{l}81 \\
(18)\end{array}$ & 85 & 3.7 & $37(8)$ & 38 & 1.2 & $\begin{array}{l}31 \\
(5)\end{array}$ & 29 & 7.2 \\
\hline
\end{tabular}

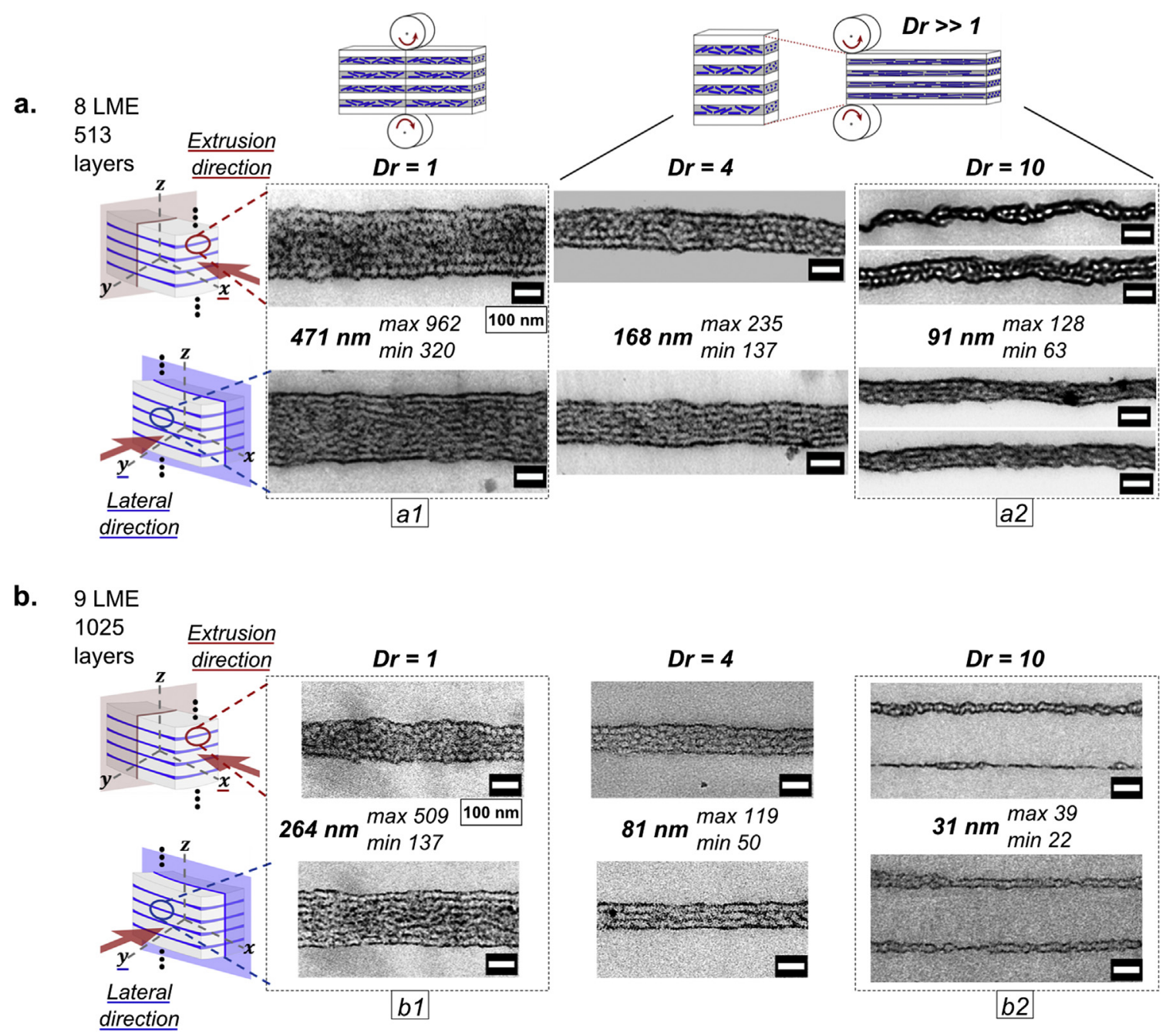

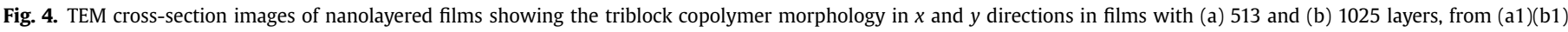

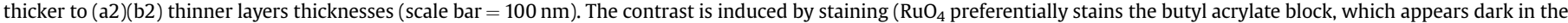
micrograph).

PMMA). This orientation decreases in the center of the layers when these layers are thick enough $(\gtrsim 100 \mathrm{~nm})$. The thickness of the confined layers and the subsequent typical number of BCPs domains within a layer can be tuned either by changing the number of LMEs and/or Dr. At high draw ratios, layers (which will eventually break up, see Fig. 4b2) containing one nanodomain can be obtained (see Fig. $4 \mathrm{a} 2$ and b2).

This relatively well-defined cylindrical morphology is fairly different from what can be observed for other as-prepared MAM samples. For example, AFM image in figure SI-4a shows the bulk morphology of a pure MAM obtained through single-screw extrusion without annealing. Though phase separation is visible, no typical morphology pattern can be identified, probably due to the short processing time $(\sim 3 \mathrm{~min})$ and the relatively low processing temperature compared to $\mathrm{T}_{\text {odt }}$. This phase separation with no clear morphology can also be observed in classically extruded (singlescrew) dry blends of PMMA and MAM, similar, but at a smaller scale, to the micro-structure of high-impact polystyrene (see figure SI-4b). MAM spin-coated thin films, when non-annealed, also display a disordered morphology (figure SI-4C).

To confirm these TEM observations, SAXS analyses were performed on the nanolayered samples in the 3 directions (Fig. 5). 
Fig. 5a1 shows typical anisotropic patterns in the $\mathrm{x}, \mathrm{y}$, and $\mathrm{z}$ directions. The concentrated intensities at the equatorial and at the pole for the TD (transverse direction) and the LD (lateral direction) patterns, respectively, show that linear objects are aligned in the flow direction. As for the ED (extrusion direction) pattern, the hexagonal (more or less circular) pattern indicates a hexagonal symmetry. This confirms the TEM observations: the block copolymer in the extruded films shows a cylindrical morphology with cylinders oriented in the extrusion flow [62]. Patterns in the z-direction show a better alignment of cylinders when the layer thickness decreases (at high draw ratios) independently of the number of layers inside the film (513 or 1025 layers obtained using 8 or 9 LME, respectively). Two well-defined spots can be observed on the equatorial line, left and right from the center beam, for thinner layers (TD of Fig. $5 \mathrm{a} 2$ and b2). These spots are less visible and more diffuse for thicker layers (TD of Fig. 5a1 and b1).

Patterns in the x-direction show a hexagonally-packed cylindrical array well defined for thicker layers, for both 8 and 9 LME films (ED of Fig. $5 \mathrm{a} 1$ and b1). Six spots separated by $60^{\circ}$ are visible with two of them located at meridional line, pointed by black arrows in the same figure. This array is less visible when the layer thickness decreases because of the confinement and the number of scattering objects in each layer falling to a lower amount (ED of Fig. $5 \mathrm{a} 2$ and b2).

Patterns in the y-direction also show a better alignment of cylinders when the layer thickness decreases. Two spots on each side of the meridional line of the center beam appear better defined for thinner layers (LD of Fig. $5 \mathrm{a} 2$ and b2) than for the thicker ones (LD of Fig. $5 a 1$ and b1). However, this effect is more pronounced for the 9 LME films because of the better alignment induced by a higher degree of confinement.

Differences between films presenting similar MAM confinement but obtained through different processing routes (by changing the number of LME and the Dr) were observed in the SAXS experiments. 1D scattering profiles of such films are showed respectively in Fig. 6a and b. Spectra of films with different processing conditions but similar layer thicknesses are plotted using the same color code (as showed in Table 1: $8 \mathrm{LME}, \mathrm{Dr}=4$ and $9 \mathrm{LME}, \mathrm{Dr}=1$ leading to layer thicknesses on the order of $200 \mathrm{~nm}$ appear in red; $8 \mathrm{LME}$, $\mathrm{Dr}=10$ and $9 \mathrm{LME}, \mathrm{Dr}=4$ leading to layer thicknesses close to $85 \mathrm{~nm}$ appear in blue).

Second order cannot be easily characterized on the SAXS spectra, even though mild shoulders around $2 q$ are more visible for thicker layers. Similar profiles have been obtained for BCP confined with nanolayer coextrusion [63]. Besides, as shown by Ruzette et al. [9], SAXS profiles of disperse MAM morphologies with curved interfaces only indicate a liquid-like ordering state of microdomains with a main scattering peak followed by a broad shoulder around $2 q$.

Nonetheless, several results can be extracted from the spectra. First, for a given BCP layer thickness, it seems that in the 1025layers films (obtained with $9 \mathrm{LME}$ ), the BCP is more sensitive to confinement. By comparing these distances for 513-layers (8 LME) and 1025-layers films at medium degree of confinement (red lines), the average cylinder-cylinder distance $(\mathrm{d}=2 \pi / q)$ values are identical for the two samples, both in lateral direction $(L D)\left(d_{L}=30 \mathrm{~nm}\right)$ and transverse direction (TD) $\left(\mathrm{d}_{\mathrm{T}}=32 \mathrm{~nm}\right)$. However, for a higher degree of confinement (blue lines), $d_{L}$ evolves when comparing 8 LME and 9 LME films, decreasing from 30 to $28 \mathrm{~nm}$ respectively for LD while $\mathrm{d}_{\mathrm{T}}$ increases from 32 to $34 \mathrm{~nm}$ for TD.

This change with Dr in both TD and LD for the 1025-layers films (9 LME) is even more pronounced at the highest degree of confinement. $d_{L}$ decreases by $16 \%$ (from 31 to $26 \mathrm{~nm}$ ) as $\mathrm{Dr}$ increases from 1 to 10 . On the contrary, $\mathrm{d}_{\mathrm{T}}$ increases by $17 \%$ (from 32 to $38 \mathrm{~nm}$ ) as Dr increases from 1 to 10 . This could be explained by a confinement effect as represented in Fig. 6c. It should be pointed out that the 8 LME films never reach these highly confined states (even at high Dr). The minimum BCP average layer thickness is only $91 \mathrm{~nm}$ at $\mathrm{Dr}=10$, whereas for the 9 LME films, it can be as low as $31 \mathrm{~nm}$ which is obviously lower than a hexagonal packing cell. This may partially explain why the peaks shift for the 9 LME films whereas they remain still for the 8 LME films. Such modifications in the packing due to confinement was observed for other BCP morphologies, such as spheres [64], cylinders [65] or lamellae [66], when confined in thin films showing terracing phenomenon. In spin-coated [65,66] or dip-coated [64] films, as-cast, after
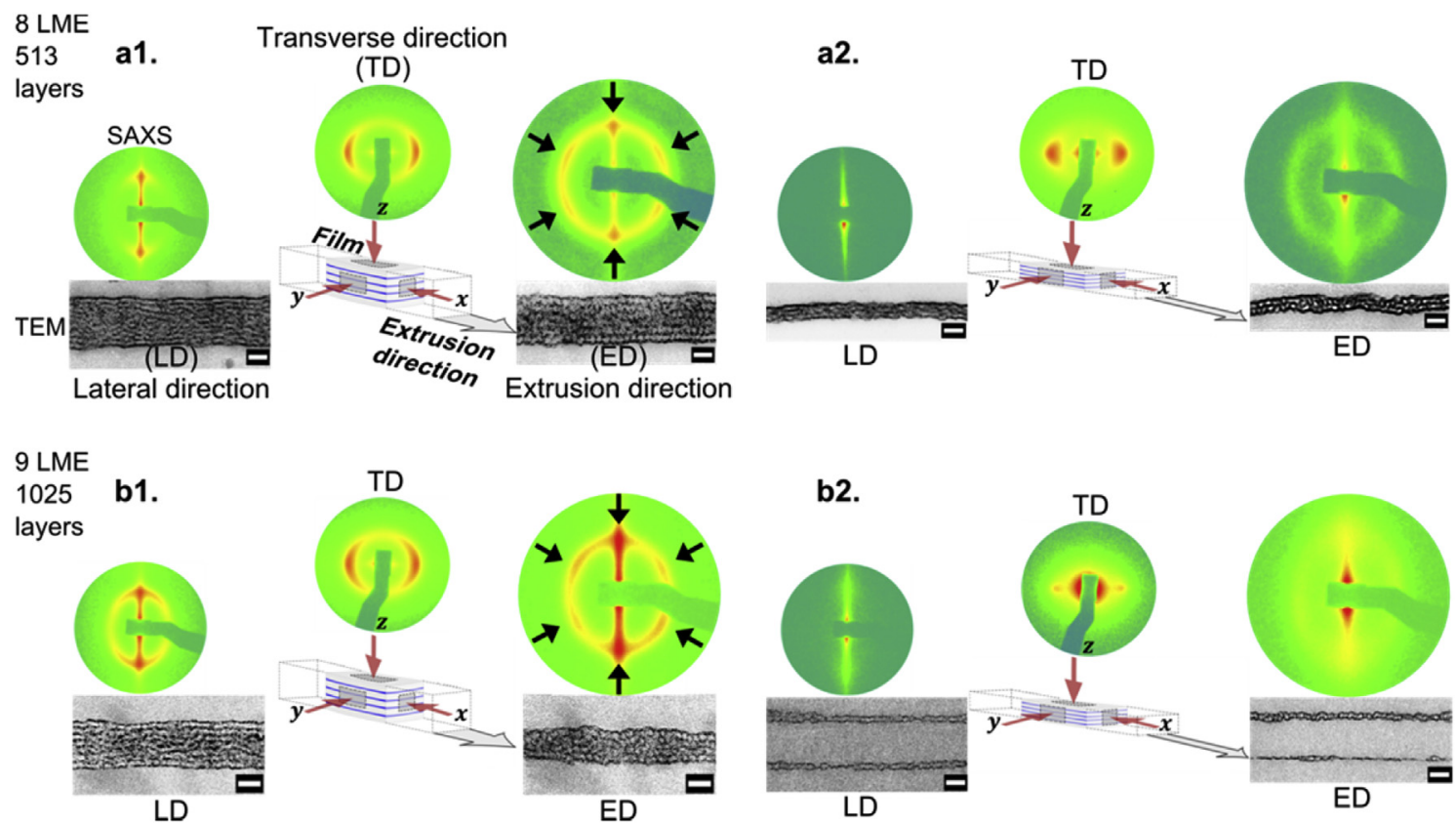

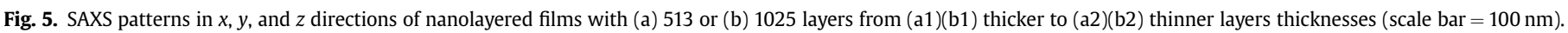


a.

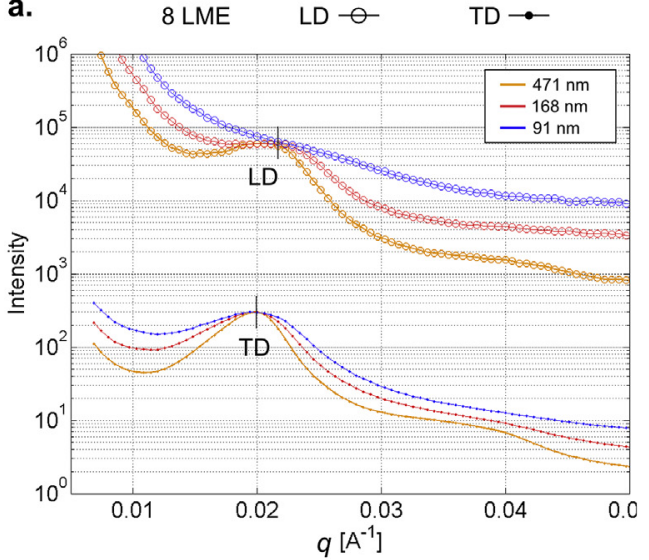

c.

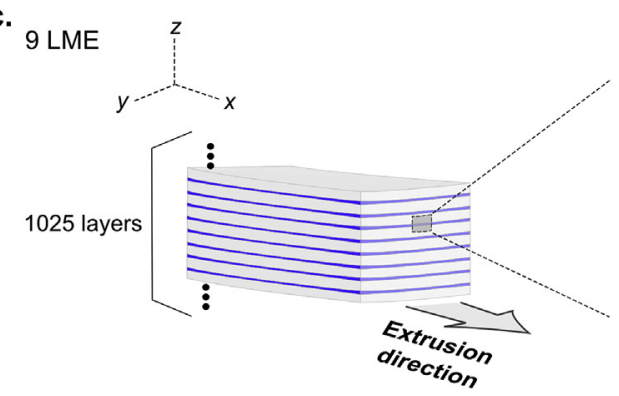

b.
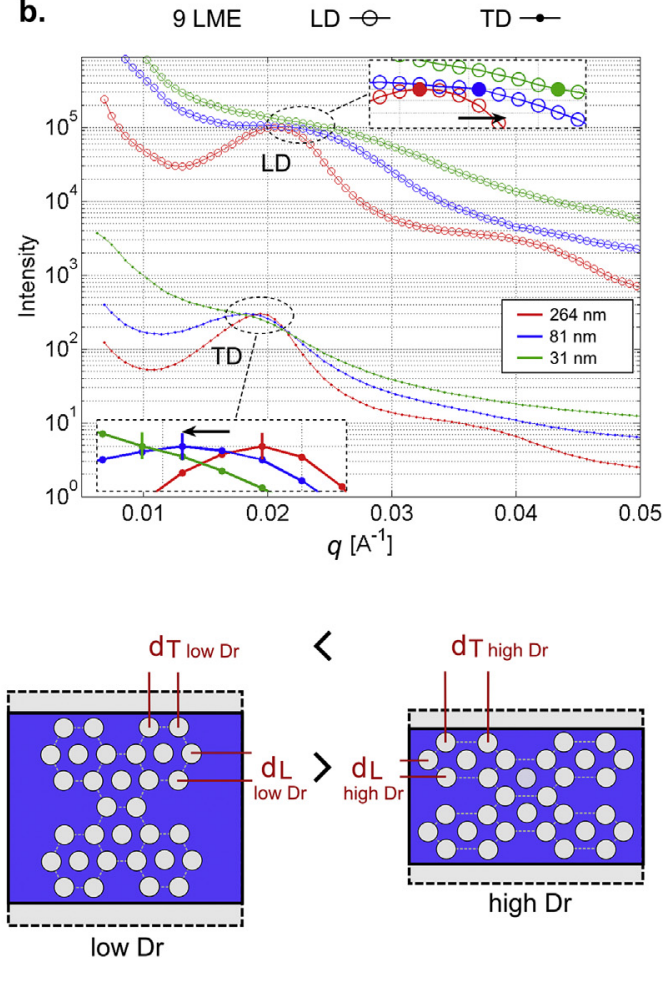

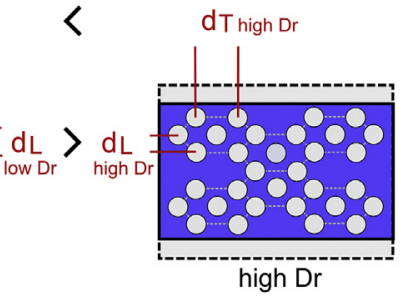

high $\mathrm{Dr}$

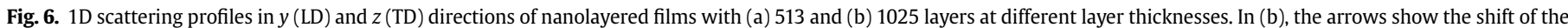

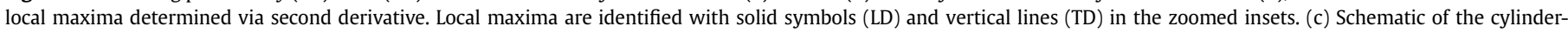
cylinder distance in a 1025-layers film.

annealing or confined between two flat solid surfaces, this phenomenon is related to the thickness of the film being incommensurate with the domain periodicity. It induces a frustration when the film thickness is slightly different from the bulk equilibrium periodicity, which causes a geometrical change in the morphology packing. As the thin film thickness is increased, this stress is released to the point where the periodic distance reaches that of the bulk [65]. Here, the morphology packing appears to be impacted by the confinement, as the BCP layer thickness decreases (i.e., the $\mathrm{Dr}$ increases), which translates into higher and lower values for $\mathrm{d}_{\mathrm{T}}$ and $\mathrm{d}_{\mathrm{L}}$, respectively.

These results show that both morphology orientation and order are influenced by the processing parameters even when similar confinement is considered. This is confirmed by measuring the Herman's orientation factor on the TD patterns, as seen in Fig. 7.

The orientation factor for a perfectly oriented cylindrical system should be 1 , and 0 for an isotropic system (see details in SI). Though a too quantitative interpretation of this analysis may be questionable (small deviations in the SAXS baseline signal may for example have an influence on the final value of the orientation factor), it is observed that the orientation factor remains almost unchanged (around 0.6) with the layer thickness for the 8 LME films, while it increases from roughly 0.4 to 0.7 as the layer thickness decreases for the 9 LME films.

It appears that the 9 LME films are somewhat more sensitive to the stretching induced by drawing than the 8 LME films. According to the thicknesses measured via AFM, the BCP layers in the 9 LME films may be closer to the BCP natural periodic length, especially at higher Dr. Nonetheless, layer instabilities more often observed with 9 LME films could result in a lower value of the orientation factor at low $\operatorname{Dr}(0.4$ vs. 0.6 for films obtained with $8 \mathrm{LME})$. But as the draw ratio increases, the combination of stretching and confinement

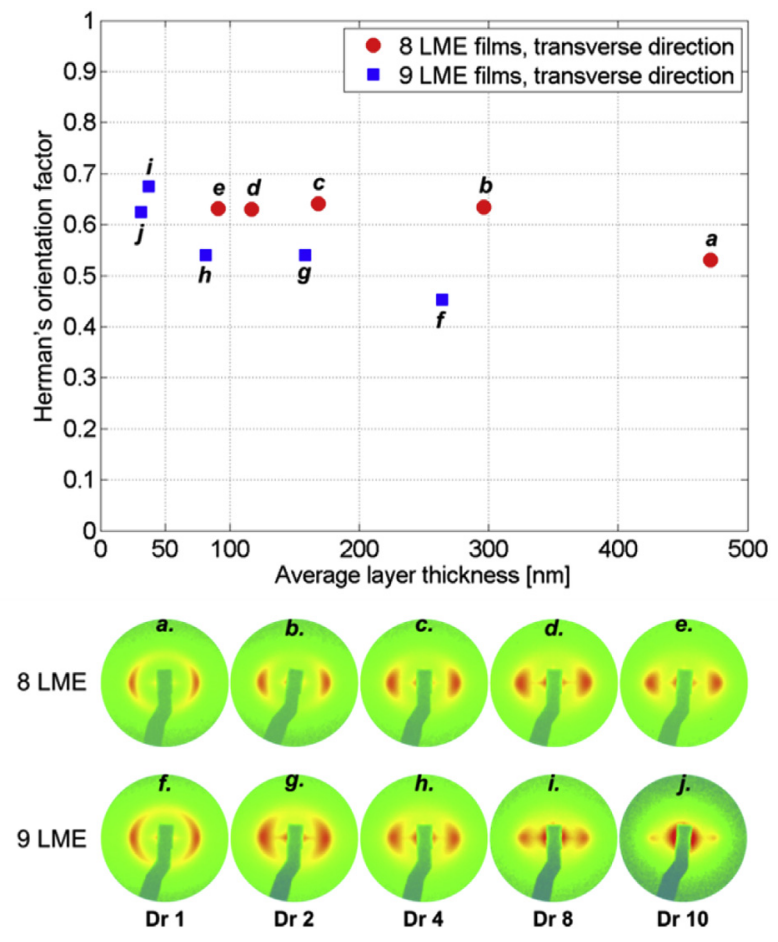

Fig. 7. Herman's orientation factor as a function of the average layer thickness for 8 and 9 LME films in the transverse direction. SAXS patterns of these films are also shown for same direction.

(more pronounced with 9 LME films) could lead to an increase in the orientation factor not observed for $8 \mathrm{LME}$ films. Such increase in 
the orientation factor as the layer thickness decreases has also been observed on PS/SEPS systems [42].

\section{Conclusion}

In this study, films containing alternated layers of a MAM triblock copolymer and PMMA were successfully extruded through nanolayer coextrusion and characterized thoroughly through AFM, TEM, and SAXS experiments. The MAM layer thickness has been successfully tuned from roughly $30 \mathrm{~nm}$ to $500 \mathrm{~nm}$, by changing the number of LME and/or Dr. Even though a lamellar morphology was characterized at equilibrium for the triblock copolymer both in bulk and thin films, a cylindrical morphology appears to be prominent in the extruded films, irrespective of the MAM layer thickness, with a mean cylinder-to-cylinder distance close to $30 \mathrm{~nm}$. We suspect the high dispersity of the copolymer, the relatively low molecular weight (the copolymer being close to its ODT) as well as the shear are responsible for favoring this cylindrical morphology over the lamellar one. Confinement via a "soft" interface (PMMA being miscible with the MAM endblocks) plays a role in the cylinders arrangement but the exact role in the morphology change remains an open question: though no clear morphology is identifiable in as extruded bulk MAM, cylinders are observed in relatively unconfined - $500 \mathrm{~nm}$ - layers in the nanolayered films. Nonetheless, the confinement induces geometric changes in the cylinder ordering, forcing the cylinders to align with the layer interface in the extrusion direction as the thickness of the layer is reduced. It is also shown that different processing routes leading to similar layer thicknesses will slightly influence the cylinder ordering as well, by deforming the hexagonal packing. We believe these results showing a control of the (as-extruded) block morphology via nanolayer coextrusion may be of interest for applications such as mechanical reinforcement of thermoplastics through the addition of BCPs with soft blocks, where no morphology control can be easily achieved via classical processing tools.

\section{Acknowledgements}

Arkema is acknowledged for providing the triblock copolymer and for giving fruitful advices concerning the sample preparation (staining) for TEM observation. Carnot Arts is acknowledged for funding the PL-GPC $220^{\circledR}$ through the "Carnot-CEQUAM project » (2010). This work has benefited from the facility and expertise of the electron microscopy facility of Institut de Biologie Paris-Seine (IBPS/FR3631 - UPMC/CNRS - Paris, France), especially G. Toutirais and G. Frebourg. We thank S. Tencé-Girault, V. Woehling and the MMC lab (UMR 7167 - ESPCI/CNRS - Paris, France) for providing access to the cryo-ultramicrotome used in the study. We thank the SOLEIL synchrotron and the SWING beamline for access to the instrumentation (project $n^{\circ}$ 20160438). More particularly, we are grateful to J. Perez (beamline manager) and T. Bizien (beamline scientist) for the help to obtain the SAXS data. We also thank V. Michel for his help with the SAXS experiments performed in the PIMM lab. We would like to acknowledge A. Guinault and A. Grandmontagne for their help with the nanolayer coextrusion setup, and C. Sollogoub, S. Bourrigaud, and L.Rubatat for fruitful discussions throughout the project.

\section{Appendix A. Supplementary data}

Supplementary data related to this article can be found at https://doi.org/10.1016/j.polymer.2017.12.044.

\section{References}

[1] C.M. Bates, F.S. Bates, 50th anniversary perspective: block polymers-pure potential, Macromolecules 50 (1) (2017) 3-22, https://doi.org/10.1021/ acs.macromol.6b02355.

[2] R.A.L. Jones, Polymers at Surfaces and Interfaces, Cambridge University Press, 1999.

[3] F.S. Bates, G.H. Fredrickson, Block copolymers-designer soft materials, Phys. Today 52 (2) (1999) 32-38, https://doi.org/10.1063/1.882522.

[4] G.H. Fredrickson, F.S. Bates, Dynamics of block copolymers: theory and experiment, Annu. Rev. Mater. Sci. 26 (1) (1996) 501-550, https://doi.org/ 10.1146/annurev.ms.26.080196.002441.

[5] I.W. Hamley, Ordering in thin films of block copolymers: fundamentals to potential applications, Prog. Polym. Sci. 34 (11) (2009) 1161-1210, https:// doi.org/10.1016/j.progpolymsci.2009.06.003.

[6] C. Park, J. Yoon, E.L. Thomas, Enabling nanotechnology with self assembled block copolymer patterns, Polymer 44 (22) (2003) 6725-6760, https: /1 doi.org/10.1016/j.polymer.2003.08.011.

[7] M.W. Matsen, Effect of architecture on the phase behavior of AB-type block copolymer melts, Macromolecules 45 (4) (2012) 2161-2165, https://doi.org/ $10.1021 / \mathrm{ma} 202782 \mathrm{~s}$.

[8] M.W. Matsen, R.B. Thompson, Equilibrium behavior of symmetric ABA triblock copolymer melts, J. Chem. Phys. 111 (15) (1999) 7139-7146, https://doi.org/ $10.1063 / 1.480006$.

[9] A.-V. Ruzette, S. Tencé-Girault, L. Leibler, F. Chauvin, D. Bertin, O. Guerret, P. Gérard, Molecular disorder and mesoscopic order in polydisperse acrylic block copolymers prepared by controlled radical polymerization, Macromolecules 39 (17) (2006) 5804-5814, https://doi.org/10.1021/ma060541u.

[10] N.A. Lynd, A.J. Meuler, M.A. Hillmyer, Polydispersity and block copolymer selfassembly, Prog. Polym. Sci. 33 (9) (2008) 875-893, https://doi.org/10.1016/ j.progpolymsci.2008.07.003.

[11] N.A. Lynd, M.A. Hillmyer, Influence of polydispersity on the self-assembly of diblock copolymers, Macromolecules 38 (21) (2005) 8803-8810, https:// doi.org/10.1021/ma051025r.

[12] N.A. Lynd, M.A. Hillmyer, Effects of polydispersity on the Order-Disorder transition in block copolymer melts, Macromolecules 40 (22) (2007) 8050-8055, https://doi.org/10.1021/ma070962r.

[13] N.A. Lynd, M.A. Hillmyer, B.J. Hamilton, The role of polydispersity in the lamellar mesophase of model diblock copolymers, J. Polym. Sci. B Polym. Phys. 45 (2007) 3386-3393, https://doi.org/10.1002/polb.21321/.

[14] M.W. Matsen, Polydispersity-induced macrophase separation in diblock copolymer melts, Phys. Rev. Lett. 99 (14) (2007) 1483041-1483044, https:// doi.org/10.1103/PhysRevLett.99.148304.

[15] M.W. Matsen, Equilibrium behavior of asymmetric ABA triblock copolymer melts, J. Chem. Phys. 113 (13) (2000) 5539-5544, https://doi.org/10.1063/ 1.1289889.

[16] W. Li, M. Liu, F. Qiu, A.-C. Shi, Phase diagram of diblock copolymers confined in thin films, J. Phys. Chem. B 117 (17) (2013) 5280-5288, https://doi.org/ 10.1021/jp309546q.

[17] H. Xiang, K. Shin, T. Kim, S. Moon, T.J. Mccarthy, T.P. Russell, The influence of confinement and curvature on the morphology of block copolymers, J. Polym. Sci. B Polym. Phys. 43 (23) (2005) 3377-3383, https://doi.org/10.1002/ polb.20641.

[18] H. Xiang, K. Shin, T. Kim, S.I. Moon, T.J. McCarthy, T.P. Russell, Block copolymers under cylindrical confinement, Macromolecules 37 (15) (2004) 5660-5664, https://doi.org/10.1021/ma049299m.

[19] C.R. Stewart-Sloan, E.L. Thomas, Interplay of symmetries of block polymers and confining geometries, Eur. Polym. J. 47 (4) (2011) 630-646, https:// doi.org/10.1016/j.eurpolymj.2010.10.013.

[20] N. Koneripalli, N. Singh, R. Levicky, F.S. Bates, P.D. Gallagher, S.K. Satija, Confined block copolymer thin films, Macromolecules 28 (8) (1995) 2897-2904, https://doi.org/10.1021/ma00112a041.

[21] T.P. Russell, Y. Chai, 50th anniversary perspective: putting the squeeze on polymers: a perspective on polymer thin films and interfaces, Macromole-

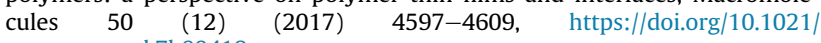
acs.macromol.7b00418.

[22] S.B. Darling, Directing the self-assembly of block copolymers, Prog. Polym. Sci. 32 (10) (2007) 1152-1204, https://doi.org/10.1016/ j.progpolymsci.2007.05.004.

[23] J. Stasiak, A.M. Squires, V. Castelletto, I.W. Hamley, G.D. Moggridge, Effect of stretching on the structure of cylinder- and sphere-forming Styrene-Isoprene-Styrene block copolymers, Macromolecules 42 (14) (2009) 5256-5265, https://doi.org/10.1021/ma8026639.

[24] G. Arya, J. Rottler, A.Z. Panagiotopoulos, D.J. Srolovitz, P.M. Chaikin, Shear ordering in thin films of spherical block copolymer, Langmuir 21 (24) (2005) 11518-11527, https://doi.org/10.1021/la0516476.

[25] A.P. Marencic, D.H. Adamson, P.M. Chaikin, R.A. Register, Shear alignment and realignment of sphere-forming and cylinder-forming block-copolymer thin films, Phys. Rev. E 81 (1) (2010) 01150301-01150310, https://doi.org/ 10.1103/PhysRevE.81.011503.

[26] K.I. Winey, S.S. Patel, R.G. Larson, H. Watanabe, Interdependence of shear deformations and block copolymer morphology, Macromolecules 26 (10) (1993) 2542-2549, https://doi.org/10.1021/ma00062a024.

[27] M. Cloitre, D. Vlassopoulos, Block copolymers in external fields: rheology, 
flow-induced phenomena, and applications, in: rianna Kontopoulou (Ed.), Applied Polymer Rheology, John Wiley \& Sons, Inc., 2011, pp. 209-239.

[28] I.W. Hamley, Structure and flow behaviour of block copolymers, J. Phys. Condens. Matter 13 (33) (2001) R643-R671, https://doi.org/10.1088/09538984/13/33/201.

[29] F.S. Bates, M.F. Schulz, A.K. Khandpur, S. Förster, J.H. Rosedale, K. Almdal, K. Mortensen, Fluctuations, conformational asymmetry and block copolymer phase behaviour, Faraday Discuss 98 (1994) 7-18, https://doi.org/10.1039/ FD9949800007.

[30] I.W. Hamley, K.A. Koppi, J.H. Rosedale, F.S. Bates, K. Almdal, K. Mortensen, Hexagonal mesophases between lamellae and cylinders in a diblock copolymer melt, Macromolecules 26 (22) (1993) 5959-5970, https://doi.org/ 10.1021/ma00074a018.

[31] R. Eskimergen, K. Mortensen, M.E. Vigild, Shear instability of a gyroid diblock copolymer, Macromolecules 38 (4) (2005) 1286-1291, https://doi.org/ $10.1021 / \mathrm{ma} 047853 \mathrm{~m}$.

[32] S. Sakurai, T. Kota, D. Isobe, S. Okamoto, K. Sakurai, T. Ono, K. Imaizumi, S. Nomura, Synchrotron small-angle x-ray scattering studies on flow-induced gyroid to cylinder transition in an elastomeric SBS triblock copolymer, J. Macromol. Sci. Phys. B 43 (1) (2004) 1-11, https://doi.org/10.1081/MB120027747.

[33] F.S. Bates, K.A. Koppi, M. Tirrell, K. Almdal, K. Mortensen, Influence of shear on the hexagonal-to-disorder transition in a diblock copolymer melt, Macromolecules 27 (20) (1994) 5934-5936, https://doi.org/10.1021/ma00098a060.

[34] K.A. Koppi, M. Tirrell, F.S. Bates, K. Almdal, K. Mortensen, Epitaxial growth and shearing of the body centered cubic phase in diblock copolymer melts, J. Rheol. 38 (4) (1994) 999-1027, https://doi.org/10.1122/1.550600.

[35] M.P. Stoykovich, M. Müller, S.O. Kim, H.H. Solak, E.W. Edwards, J.J. de Pablo, P.F. Nealey, Directed assembly of block copolymer blends into nonregular device-oriented structures, Science 308 (5727) (2005) 1442-1446, https:// doi.org/10.1126/science.1111041.

[36] A.P. Marencic, R.A. Register, Controlling order in block copolymer thin films for nanopatterning applications, Annu. Rev. Chem. Biomol. Eng. 1 (2010) 277-297, https://doi.org/10.1146/annurev-chembioeng-073009-101007.

[37] J. Bang, U. Jeong, D.Y. Ryu, T.P. Russell, C.J. Hawker, Block copolymer nanolithography: translation of molecular level control to nanoscale patterns, Adv. Mater. 21 (47) (2009) 4769-4792, https://doi.org/10.1002/adma.200803302.

[38] J.M. Carr, D.S. Langhe, M.T. Ponting, A. Hiltner, E. Baer, Confined crystallization in polymer nanolayered films: a review, J. Mater. Res. 27 (10) (2012) 1326-1350, https://doi.org/10.1557/jmr.2012.17.

[39] M. Ponting, T.M. Burt, L.T.J. Korley, J. Andrews, A. Hiltner, E. Baer, Gradient multilayer films by forced assembly coextrusion, Ind. Eng. Chem. Res. 49 (23) (2010) 12111-12118, https://doi.org/10.1021/ie100321h.

[40] M. Ponting, A. Hiltner, E. Baer, Polymer nanostructures by forced assembly: process, structure, and properties, Macromol. Symp. 294 (1) (2010) 19-32, https://doi.org/10.1002/masy.201050803.

[41] R.Y.F. Liu, Y. Jin, A. Hiltner, E. Baer, Probing nanoscale polymer interactions by forced-assembly, Macromol. Rapid Commun. 24 (16) (2003) 943-948, https: / doi.org/10.1002/marc.200300051.

[42] T.M. Burt, J. Keum, A. Hiltner, E. Baer, L.T.J. Korley, Confinement of elastomeric block copolymers via forced assembly coextrusion, ACS Appl. Mater. Interfaces 3 (12) (2011) 4804-4811, https://doi.org/10.1021/am201297f.

[43] T.M. Burt, A.M. Jordan, L.T.J. Korley, Toward anisotropic materials via forced assembly coextrusion, ACS Appl. Mater. Interfaces 4 (10) (2012) 5155-5161, https://doi.org/10.1021/am301072s.

[44] T.M. Burt, A.M. Jordan, L.T.J. Korley, Investigating interfacial contributions on the layer-thickness-dependent mechanical response of confined selfassembly via forced assembly, Macromol. Chem. Phys. 214 (8) (2013) 873-881, https://doi.org/10.1002/macp.201200588.

[45] It is unclear if this is by the use of a sacrificial layer or by changing the speed of the chill rolls at the exit die, i.e. increasing the draw ratio.

[46] T.M. Burt, S. Monemian, A.M. Jordan, L.T.J. Korley, Thin film confinement of a spherical block copolymer via forced assembly Co-Extrusion, Soft Matter 9 (17) (2013) 4381-4385, https://doi.org/10.1039/C3SM27797F.

[47] A. Bironeau, T. Salez, G. Miquelard-Garnier, C. Sollogoub, Existence of a critical layer thickness in PS/PMMA nanolayered films, Macromolecules 50 (10) (2017) 4064-4073, https://doi.org/10.1021/acs.macromol.7b00176.

[48] M. Boufarguine, A. Guinault, G. Miquelard-Garnier, C. Sollogoub, PLA/PHBV films with improved mechanical and gas barrier properties, Macromol. Mater. Eng. 298 (10) (2013) 1065-1073, https://doi.org/10.1002/mame.201200285.

[49] X. Li, G. Miquelard-Garnier, A. Guinault, C. Sollogoub, G. Regnier, A. Rozanski, Forced assembly by multilayer coextrusion to create oriented graphene reinforced polymer nanocomposites, Polymer 55 (1) (2013) 248-257, https: / doi.org/10.1016/j.polymer.2013.11.025.

[50] S. Fernandes Nassar, A. Guinault, N. Delpouve, V. Divry, V. Ducruet, C. Sollogoub, S. Domenek, Multi-scale analysis of the impact of polylactide morphology on gas barrier properties, Polymer 108 (2017) 163-172, https:/ doi.org/10.1016/j.polymer.2016.11.047.

[51] S. Roland, G. Miquelard-Garnier, M. Gervais, A. Guinault, C. Sollogoub, Controlling the order of triblock copolymer via confinement induced by forced self-assembly, Mater. Today Commun (2016) 37-43, https://doi.org/10.1016 j.mtcomm.2015.11.003. No. 6.

[52] J.S. Trent, J.I. Scheinbeim, P.R. Couchman, Ruthenium tetraoxide staining of polymers for electron microscopy, Macromolecules 16 (4) (1983) 589-598, https://doi.org/10.1021/ma00238a021.

[53] R. Vitali, E. Montani, Ruthenium tetroxide as a staining agent for unsaturated and saturated polymers, Polymer 21 (10) (1980) 1220-1222, https://doi.org 10.1016/0032-3861(80)90091-9.

[54] G. David, J. Pérez, Combined sampler robot and high-performance liquid chromatography: a fully automated system for biological small-angle x-ray scattering experiments at the synchrotron SOLEIL SWING beamline, J. Appl. Crystallogr. 42 (5) (2009) 892-900, https://doi.org/10.1107/ S0021889809029288.

[55] S.O. Kim, B.H. Kim, K. Kim, C.M. Koo, M.P. Stoykovich, P.F. Nealey, H.H. Solak, Defect structure in thin films of a lamellar block copolymer self-assembled on neutral homogeneous and chemically nanopatterned surfaces, Macromolecules 39 (16) (2006) 5466-5470, https://doi.org/10.1021/ma060087u.

[56] E. Huang, P. Mansky, T.P. Russell, C. Harrison, P.M. Chaikin, R.A. Register C.J. Hawker, J. Mays, Mixed lamellar Films: evolution, commensurability ef fects, and preferential defect formation, Macromolecules 33 (1) (2000) 80-88, https://doi.org/10.1021/ma9912711.

[57] W. Li, M. Müller, Defects in the self-assembly of block copolymers and thei relevance for directed self-assembly, Annu. Rev. Chem. Biomol. Eng. 6 (1) (2015) 187-216, https://doi.org/10.1146/annurev-chembioeng-061114 123209.

[58] S.W. Sides, G.H. Fredrickson, Continuous polydispersity in a self-consistent field theory for diblock copolymers, J. Chem. Phys. 121 (10) (2004) 4974-4986, https://doi.org/10.1063/1.1776557.

[59] V. Khanna, E.W. Cochran, A. Hexemer, G.E. Stein, G.H. Fredrickson, E.J. Kramer, X. Li, J. Wang, S.F. Hahn, Effect of chain architecture and surface energies on the ordering behavior of lamellar and cylinder forming block copolymers, Macromolecules 39 (26) (2006) 9346-9356, https://doi.org/10.1021/ ma0609228.

[60] A. Bironeau, J. Dirrenberger, C. Sollogoub, G. Miquelard-Garnier, S. Roland Evaluation of morphological representative sample sizes for nanolayered polymer blends, J. Microsc. 264 (1) (2016) 48-58, https://doi.org/10.1111/ jmi.12415.

[61] K. Mortensen, M.E. Vigild, Reinvestigation of the block copolymer modulated lamellar structure, Macromolecules 42 (5) (2009) 1685-1690, https://doi.org/ $10.1021 / \mathrm{ma} 8024249$.

[62] M.J. Folkes, A. Keller, F.P. Scalisi, An extrusion technique for the preparation o "single-Crystals" of block copolymers, Colloid Polym. Sci. - Colloid Polym Sci 251 (1973) 1-4, https://doi.org/10.1007/BF01501413.

[63] J.H. Chu, P. Rangarajan, J.L. Adams, R.A. Register, Morphologies of strongly segregated polystyrene-poly(dimethylsiloxane) diblock copolymers, Polyme 36 (8) (1995) 1569-1575, https://doi.org/10.1016/0032-3861(95)99001-B.

[64] S. Roland, D. Gaspard, R.E. Prud'homme, C.G. Bazuin, Morphology evolution in slowly dip-coated supramolecular PS-B-P4VP thin films, Macromolecules 45 (13) (2012) 5463-5476, https://doi.org/10.1021/ma3007398.

[65] A. Knoll, L. Tsarkova, G. Krausch, Nanoscaling of microdomain spacings in thin films of cylinder-forming block copolymers, Nano Lett. 7 (3) (2007) 843-846, https://doi.org/10.1021/nl070006n.

66] P. Lambooy, T.P. Russell, G.J. Kellogg, A.M. Mayes, P.D. Gallagher, S.K. Satija, Observed frustration in confined block copolymers, Phys. Rev. Lett. 72 (18) (1994) 2899-2902, https://doi.org/10.1103/PhysRevLett.72.2899. 